\title{
Aftereffect and Accommodation Anisotropy in Metal- Particle and Metal-Evaporated Recording Media
}

\author{
Lawrence H, Bennett ${ }^{1,2}$, Lydon J. Swartzendruber ${ }^{1}$, Ferenc Vajda ${ }^{2,3}$, Edward Della Torre ${ }^{2}$, and Jack H. Judy ${ }^{4}$ \\ ${ }^{1}$ National Institute of Standards and Technology, Gaithersburg, MD 20899 \\ ${ }^{2}$ Institute for Magnetics Research, The George Washington University, Washington, D.C. 20052 \\ ${ }^{3}$ Digital Measurement Systems, Inc., Burlington, MA 01803 \\ ${ }^{4}$ The Center of Micromagnetics and Information Technology (MINT) \\ Department of Electrical Engineering, University of Minnesota, Minneapolis, MN 55455
}

\begin{abstract}
Aftereffect and accommodation are measured in metal recording media using either evaporated cobalt (ME) or particulate iron (MP) as the magnetic material. These materials are highly anisotropic and measurements have been made parallel to the plane of the media both along the easy direction and along the hard direction. Along the easy direction, the loops are square and there is only a small difference between the coercive field, $H_{c}$, and the remanent coercive field, $H_{c i}$. Along the hard direction the squareness is approximately one-half that along the easy axis and $H_{c i}$ is shifted considerably from $\boldsymbol{H}_{\boldsymbol{c}}$.
\end{abstract}

\section{INTRODUCTION}

Aftereffect is a time drift in the magnetization occurring after the magnetic field has been rapidly switched and is due to thermal activation over an energy barrier[1-2]. Accommodation (also called reptation [1]) is the drift of a minor loop that occurs when the minor loop is repeatedly cycled and is due to changes in the interaction field that occur during the cycling and has been modeled using physically realistic Preisach-based scalar accommodation models [3-7]. Both effects are important considerations in high-density recording media. As previously discussed [8,9], broad distributions in the switching fields, and/or in the interaction fields, can cause the aftereffect to follow a $\log$ (time) behavior and accommodation to follow a $\log$ (number of cycles) behavior. In particular, the magnetization decay for the aftereffect rate can be expressed in terms of a decay rate $S$, where the magnetization, $M$, is given by $M=M_{0}+S M_{s} \ln (t)$, where $M_{s}$ is the saturation magnetization. In this form $S$ is dimensionless and will be given as a percent.

When the magnetic field is rapidly switched, the magnitude of the aftereffect will depend on the initial and final magnetic field. Of greatest interest is the value of $S$ measured when the field is switched from a positive value, high enough to saturate the magnetization, to a negative field. It has been often stated that $S$ has its greatest value for negative fields near the coercive field. However, for the case of a recording media utilizing

Manuscript received February 4, 1997

Lawrence H. Bennett, 703-729-8299, fax 703-729-8251,

bennett@seasva.gwu.edu

Lydon J. Swartzendruber, lydon@nist.gov

Ferenc Vajda, ferenc_vajda@dms-magnetics.com

Edward Della Torre, dellator@seas.gwu.edu

Jack H. Judy, judy@ee.umn.edu $\gamma \mathrm{Fe}_{2} \mathrm{O}_{3}$ particles [10] and for one using $\mathrm{CrO} 2$ [11], $S$ has the largest magnitude for a negative field closer to the remanent coercive field $\left(H_{c i}\right)$ rather than to the coercive field $\left(H_{c}\right)$. This was also true for the magnitude of the accommodation [10].. Here we measure aftereffect and accommodation in two commercially produced recording media, fabricated using evaporated cobalt (ME) or particulate iron (MP) as the magnetic material. As fabricated, these materials are highly anisotropic. The MP tape has a layer of fine, elongated, iron metal and thickness of about $2 \mu \mathrm{m}$. The ME tape has curved Co grains formed at an angle to the substrate and a thickness of about $0.2 \mu \mathrm{m}$. We have measured the aftereffect parallel to the plane of the media both along the easy direction and along the hard direction. Along the easy direction, the loops are square and there is only a small difference between $H_{c}$ and $H_{c i}$. Along the hard direction the squareness is approximately one-half that along the easy axis and the remanent coercive fields is shifted considerably from the coercive field.

We find that, as for the previous results for $\mathrm{\gamma Fe}_{2} \mathrm{O}_{3}$ material, the maximum aftereffect decay rate for the MP tape occurs when the field is switched to a value near the remanent coercive field. However for the ME tape, the maximum aftereffect decay rate occurs near the coercive field rather than near the remanent coercive field. Further, in contrast with the MP tape, the remanent coercivity in the hard direction of the ME tape is less than that in the easy direction.

\section{EXPERIMENTAL}

Anisotropy measurements are displayed in Figs. 1 and 2. To obtain these data, an angle was set and then a field large enough to saturate the material was applied. The field was then reduced to zero and the remanent magnetizations parallel to the applied field, $M_{r x}$, and perpendicular to the applied field, $M_{r y}$, were measured. A value for the coercive field, $H_{c}$, was then determined. The values of $M_{r x}$ and $H_{c}$ are a maximum in the easy direction and a minimum in the hard direction. The value of $M_{r y}$ is zero in both the easy and hard directions. It can be seen from Figs 1 and 2 that (1) both tapes are highly anisotropic, (2) the moment per unit area of the ME is about one-tenth that of the MP tape, (3) both tapes have directions in which $M_{r x}$ and $M_{r y}$ are of equal magnitudes, and (4) the ME tape has a greater reduction in coercive field in the hard direction than does the MP tape. The sample shape was circular, hence the observed anisotropy is due to the 


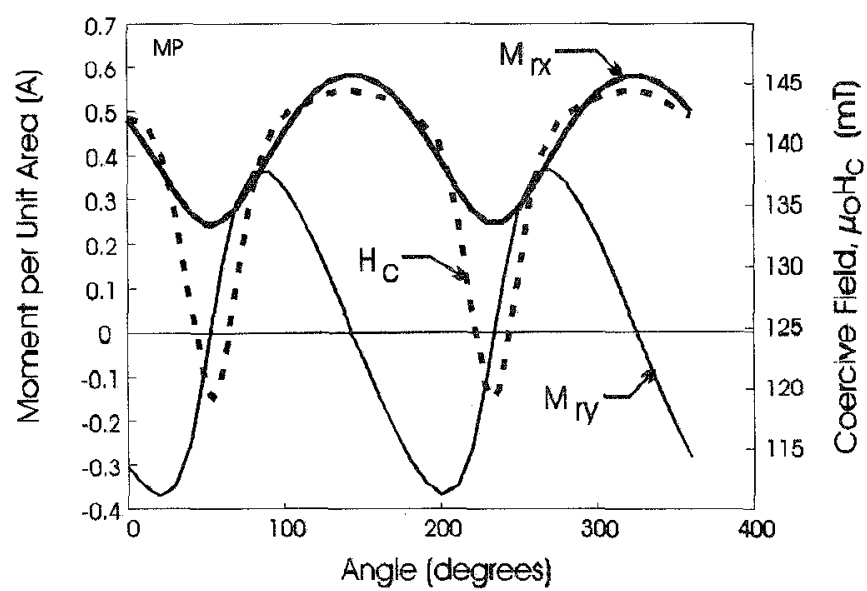

Fig. 1. Mangetization remanances, $\mathrm{M}_{\mathrm{rx}}$ and $\mathrm{M}_{\mathrm{ry}}$ (left axis) and coercive field (right axis) as a function of orientation of the MP tape.

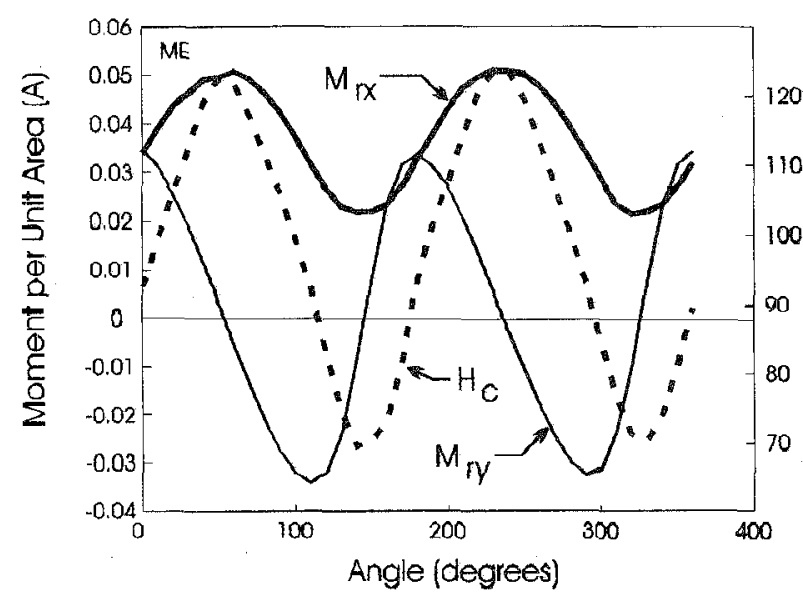

Fig. 2. Magnetization remanances, $M_{\mathrm{rx}}$ and $\mathrm{M}_{\mathrm{ry}}$ (left axis) and coercive field (right axis) as a function of orientation of the ME tape.

microstructure of the tape.

Hysteresis loops in the easy and hard directions for the MP tape are shown in Fig. 3, and for the ME tape in Fig.4. For the MP tape the squareness of the loop along the easy direction is about 0.8 , and along the hard direction about 0.4 . The squareness of the ME tape is slightly greater in the easy direction, and slightly less in the hard direction.

The remanent loops taken at negative fields are plotted, along with portions of the hysteresis loops, in Figs. 5 and 6. The remanent loop magnetization (shown on the y-axes in Figs. 5 and 6 ) is obtained by first saturating the sample in the positive direction, then changing to the field indicated on the $x$ axes, then relaxing the field to zero and measuring the resultant magnetization. The remanent coercive field, $H_{c i}$ is that field for which the magnetization becomes zero at zero field. For the MP tape material we have, along the easy axis, $H_{c}=-140 \mathrm{mT}$ and $H_{c i}=-150 \mathrm{mT}$, and along the hard direction we find $H_{c}=$ $110 \mathrm{mT}$ and $H_{c i}=-180 \mathrm{mT}$. For the ME tape along the easy axis, $H_{c}=-115 \mathrm{mT}$ and $H_{c i}=-125 \mathrm{mT}$, and along the hard direction $H_{c}=-70 \mathrm{mT}$ and $H_{c i}=-97 \mathrm{mT}$. An interesting difference between the two materials is that, for the MP tape, $H_{c i}$ is larger in the hard direction, whereas, for the ME tape, it is larger in the easy direction.

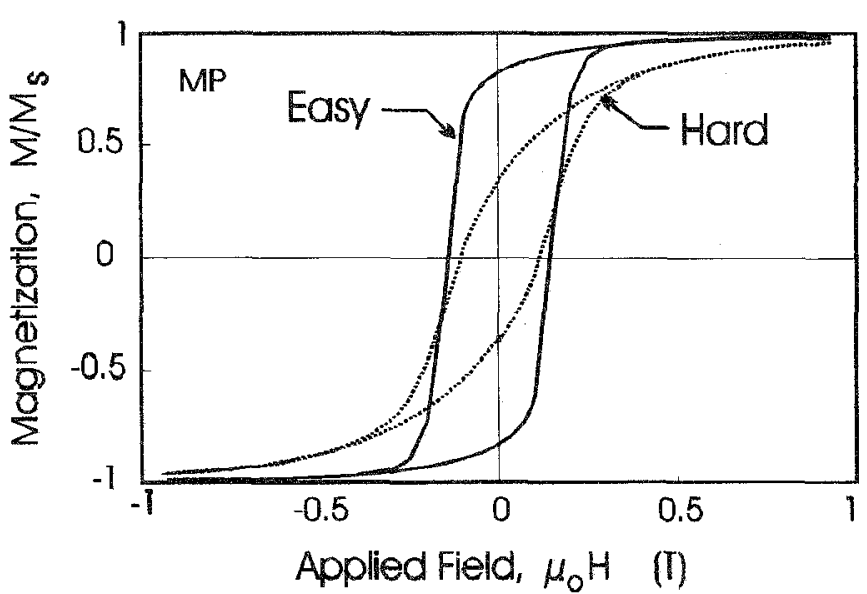

Fig. 3. Hysteresis loops in the easy and hard directions (in plane) for the MP tape.

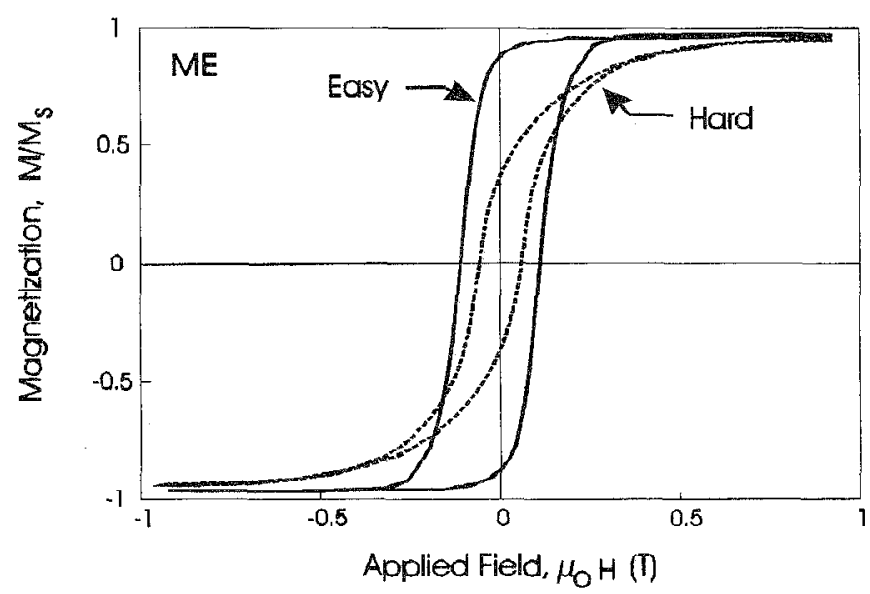

Fig. 4. Hysteresis loops in the easy and hard directions (in plane) for the ME tape.

The aftereffect decay rates in the easy and hard directions for the MP tape are shown in Fig.7, and for the ME tape in Fig. 8. The decay rates are determined by first saturating the material with a positive field, $\mathrm{H}_{1}$, then rapidly switching to a negative field, $\mathrm{H}_{2}$, (plotted along the $x$ axes in Figs 7 and 8), and then measuring the magnetization as a function of time. The value of $M / M_{s}$, where $M_{s}$ is the saturation magnetization, is then plotted vs. the natural logarithm of time. This plot is a straight line and its slope, $S$, is termed the decay rate. Determined in this way, $S$ is dimensionless and is plotted as a percent. $S$ is a function of $\mathrm{H}_{2}$, the direction of $\mathrm{H}$ (both $\mathrm{H}_{1}$ and $\mathrm{H}_{2}$ were always in the same direction for these measurements).

\section{DisCUsSION}

For both materials, there is little difference between $H_{c}$ and $H_{c i}$ along the easy axis (see Figs. 7 and 8). Along the easy axes, $S$ is largest for values of $\mathrm{H}_{2}$ approximately equal to $H_{c}$ and $H_{c i}$. For the MP tape in the hard direction, where there is a large difference between $H_{c}$ and $H_{c i}$, the largest $S$ value occurs nearest $H_{c i}$. This behavior is the same as that observed previously [10] for a recording medium fabricated with $\gamma \mathrm{Fe}_{2} \mathrm{O}_{3}$ particles. However, for the $\mathrm{ME}$ tape in the hard direction, where there is also a distinct difference between the 


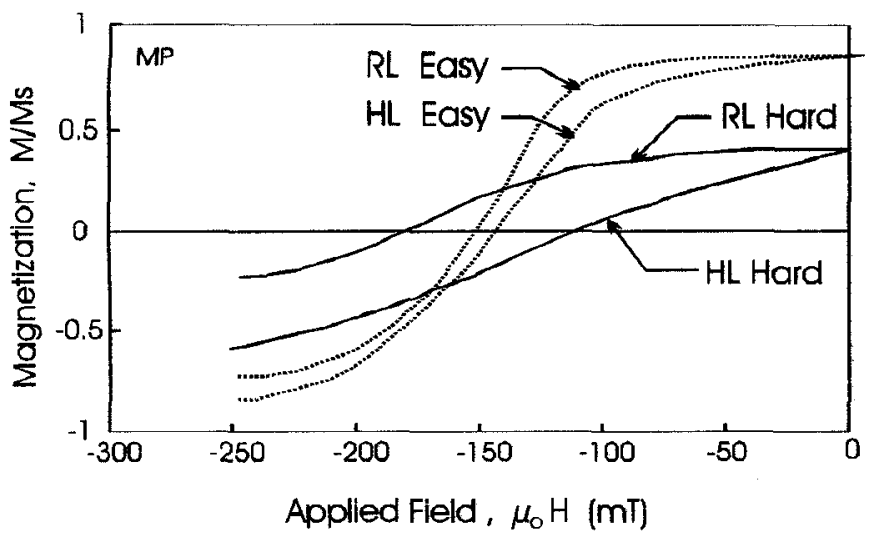

Fig. 5 Remanence loops (RL) and portions of the hysteresis loops (HL) in the easy and hard directions for the MP tape.

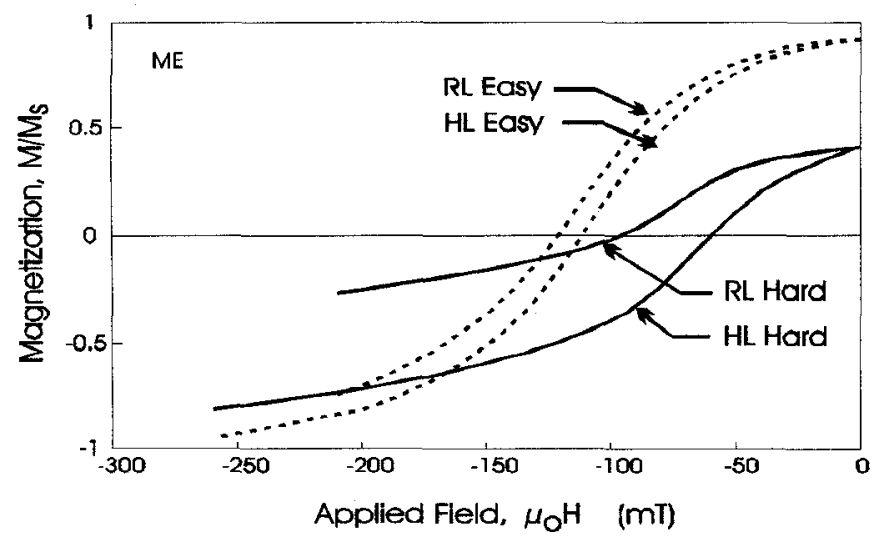

Fig. 6. Remanance loops (RL) and portions of the hysteresis loops (HL) in the easy and hard directions for the ME tape.

values of $H_{c}$ and $H_{c i}$, the maximum in the decay rate occurs near $H_{c}$ rather than near $H_{c i}$.

The $S$ values in the easy direction for the MP material are a factor of 3 larger than those found previously [10] for the $\gamma \mathrm{Fe}_{2} \mathrm{O}_{3}$ medium. For the $\mathrm{ME}$ tape the maximum magnitudes of $S$ are about $30 \%$ greater than found in the MP tape. However, in contrast with the MP tape, the remanent coercivity in the hard direction of the ME tape is less than that in the easy direction.

The accommodation behavior of the MP tape(see Fig. 8) is qualitatively similar to that found previously [10] for a $\gamma \mathrm{Fe}_{2} \mathrm{O}_{3}$ recording medium. The largest accommodation and aftereffect occur for values of $\mathrm{H}_{2}$ near the remanent coercive field. The accommodation observed in the ME tape appears to be qualitatively different than in the MP tape, in that the maximum accommodation and aftereffect are less than the remanent coercive field. In fact, the accommodation for the ME tape appears largest for $\mathrm{H}_{2}$ less than the coercive field. The origin of these differences is not clear at this time. Theoretical explanations are current under development.

\section{ACKNOWLEDGMENTS}

We thank R.D. McMichael for helpful discussions, R.V. Drew, H.J. Brown, and D. Mathews for technical assistance, and. Members of the Institute for Magnetics for useful advice.

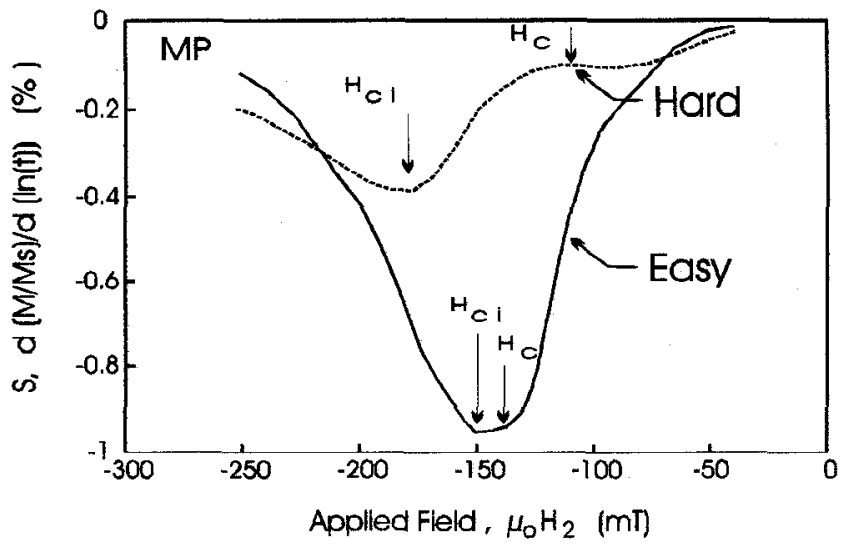

Fig. 7. Aftereffect decay rates for the MP tape in the easy and hard directions as a function of the field applied after first saturating with a positive field. Arrows mark the location of the coercive, $H_{c}$, and remanent coercive, $H_{c i}$, fields.

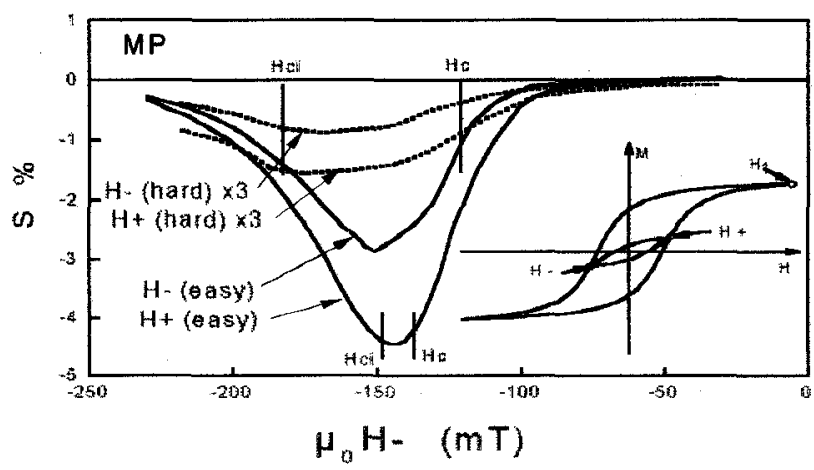

Fig. 8. Accommodation rates for the MP tape. After saturation at $\mathrm{H}_{1}$ and field change to $\mathrm{H}-$, the loop is cycled between $\mathrm{H}-$ and $\mathrm{H}+$.

\section{REFERENCES}

[1] M. El-Hilo, K. O'Grady, and R.W. Chantrell, "Time dependence effects and reptation measurements" IEEE Trans. Magn., vol. 29, pp. 3664-3666, (1993).

[2] V.G. Lewis, P.I. Mayo, and K. O'Grady, "Reptation effects in particulate systems. II Experimental studies" J. Appl. Phys 73, pp. 6656-6658 (1993).

[3] E. Della Torre, "A Preisach model for accommodation" IEEE Trans. Magn., vol 30, pp 2701-2707 (1994).

[4] F. Vajda and E. Della Torre, "Identification of parameters in an accommodation model" IEEE Trans. Magn. vol. 30, pp. 4371-4373 (1994).

[5] E. Della Torre and F. Vajda, "Properties of Accommodation Models" IEEE Trans. Magn., vol. 31, pp 1775-1780 (1995).

[6] E. Della Torre, "Effect of interaction on the magnetization of single domain particles" IEEE Trans. Audio Electroacous. vol. 14, pp. 86-93 (1996).

[7] E. Della Torre and F. Vajda, "Physical foundation for parameter identification in magnetic materials (invited)" presented at INTERMAG '96, submitted to IEEE Trans. Magn.

[8] M.P. Sharrock, "Time dependence of switching fields in magnetic recording media" J. Appl. Phys. vol 76 pp 6413-6418 (1994).

[9] E. Dan Dahlberg, D.K. Lottis, R.M. White, M. Matson, and E. Engle, "Ubiquitous nonexponential decay: the effect of long-range coupling?" $J$. Appl. Phys. vol 76 pp. 6396-6400 (1994).

[10] L.J. Swartzendruber, L.H. Bennett, F. Vajda, and E, Della Torre,"Relationship between the measurement of accommodation and aftereffect" Physica $B$, in press.

[11] S.Uren,K. O'Grady, and R.W. Chantrell, "Magnetic viscosity effects in digital recording media" J. de PhysiqueC8 S12 vol. 49 pp. 1927-1928 (1988). 